\title{
Understanding the Emergence of Behavior Problems in Young Children With Developmental Delays
}

\author{
Keith Crnic, PhD; Casey Hoffman, PhD; Catherine Gaze, PhD; \\ Craig Edelbrock, PhD
}

\begin{abstract}
Children with developmental delays have a much higher incidence of behavior problems than do children who are typically developing. This article reviews the current research on the occurrence and nature of behavior problems in these children, with particular attention to issues relevant to young children and the type and severity of problems that have been observed at ages as young as 2 years. Evidence in support of a conceptual framework for understanding how such behavior problems may develop is presented, with a particular focus on early biological or constitutional factors, family stress, and children's developing self-regulatory capacities. Implications for practice are discussed. Key words: behavior problems, developmental delay, families, self-regulation
\end{abstract}

$\mathbf{I}$ N RECENT YEARS, there has been a good deal of attention focused on the occurrence of behavior problems in older children and adolescents with developmental disabilities, including those with mental retardation. From this work, there have emerged strong indications that children with developmental problems may have up to 4 times the likelihood of having a concurrent mental health diagnosis compared with children who are typically developing (Baker, Blacher, Crnic, \& Edelbrock, 2002; Pfeiffer \& Baker, 1994). Despite this accruing knowledge in older children, there is very little known about the presence of behavior problems in young children with devel-

From the Penn State University, University Park, Pa.

This work was supported in part by grant \#HD34879 from the National Institute of Child Health and Human Development, and addresses efforts of the Collaborative Family Study (Keith Crnic, principal investigator; and Drs Bruce Baker,Jan Blacher, and Craig Edelbrock, coinvestigators).

Corresponding author: Keith Crnic, PbD, Department of Psychology, 417 Moore Bldg, Penn State University, University Park, PA 16802 (e-mail: kac8@psu.edu). opmental (cognitive) delays, and how these problems emerge during the early childhood period.

The co-occurrence of mental retardation and mental health problems is often referred to as "dual diagnosis," and it is only within the past 2 decades that concerns associated with dual diagnosis have developed within the field (Borthwick-Duffy, 1994; Matson \& Frame, 1986). For many years, clinicians did not specifically attend to the variety of symptoms of psychopathology in persons with mental retardation or in young children with developmental delays. In all likelihood, this lack of attention was the result of "diagnostic overshadowing," a process in which deficits related primarily to intellectual limitations dominated clinical concerns, and the symptoms consistent with criteria for psychological disorders were often considered to be a function of the intellectual deficit rather than indicative of another diagnosis (BorthwickDuffy, 1994). The concept of dual diagnosis, however, avoids labeling either the developmental disability or the psychological disorder as primary, and reflects the belief that the diagnoses are distinct and equally important 
to recognize for treatment (Pfeiffer \& Baker, 1994).

In this article, we review the current status of research on behavior problems in children with early developmental delays or disabilities. We then consider a model that attempts to explain the emergence of behavior problems in these children, and discuss evidence in support of the major constructs involved. Finally, we consider the implications of these issues for practice.

\section{BEHAVIOR PROBLEMS AND EARLY DEVELOPMENTAL DELAY}

\section{General considerations in dual diagnosis}

Before addressing how behavior problems may develop in young children with developmental delays, it is useful to provide some background on the key issues associated with behavior problems in children and adolescents with developmental delays that set the stage for understanding how such problems may develop. Prevalence in the population as well as the descriptive data on the types of behavior disorders typically associated with developmental delay are especially important concerns for clinicians with interests in this population of children. The prevalence of psychopathology reported in the literature varies considerably, ranging from low estimates of less than $10 \%$ to high estimates of more than $80 \%$ across age groups (BorthwickDuffy, 1994; Nezu, Nezu, \& Gill-Weiss, 1992). Not only is there a wide range in the estimates of the numbers of individuals who might be dually diagnosed, but research also indicates that there is a wide range in the types of psychological disorders that may be present (Dykens, 2000; Jacobson, 1990).

The disparate prevalence estimates reported in the literature can be traced to several factors (for review see Borthwick-Duffy, 1994). Perhaps the most important consideration is that the classification of mental retardation or developmental delay across research studies varies widely. Typically, definitions of mental retardation or meaningful developmental delay include scores on standardized cognitive assessments that reflect 2 or more standard deviations below the mean as well as deficits in adaptive behavior. Although more recent research studies use both criteria, some studies use only the standardized cognitive criterion. To further complicate matters, basic definitions of mental retardation and developmental delay have changed over time, as have definitions or classification systems for psychopathology.

Across the childhood period, research indicates that the presence of behavior problems in children with developmental problems is substantial. For example, Jacobson (1990) reported a prevalence of $40 \%$ in children aged 5 to 17 years, with a wide range of diagnoses apparent. In a study of children with mental retardation and epilepsy, Steffenburg, Gillberg, and Steffenburg (1996) also found high rates of psychiatric diagnoses. Within the population of children diagnosed with mild mental retardation, the most common diagnoses were autism and autism-like disorders. Eighteen percent of the children with mild mental retardation were diagnosed with attentiondeficit hyperactivity disorder (ADHD).

A recent epidemiological study of psychopathology in children with cognitive delays (Stromme \& Diseth, 2000) identified all of the children born within a 5-year period in a county in Norway who met criteria for mental retardation. The presence of a psychiatric diagnosis was evaluated based on a semi-structured interview with the parents and a clinical child interview, both of which were modeled on the International Statistical Classification of Diseases, 10th Revision classification system. Psychiatric diagnosis was then classified as either comorbid features of the developmental disorder or a predominant psychiatric disorder. Stromme and Diseth found that overall $37 \%$ of the sample met criteria for psychiatric disorders. The vast majority of these diagnoses (74\%) were considered comorbid to the syndrome, while the remaining were considered predominantly psychiatric in origin. Of those that were psychiatric, the problems were evenly split 
between autism and hyperkinesis (ADHD), corroborating the findings of Steffenburg et al. (1996). It seems apparent from these general studies across childhood that behavior problems in this population are a major concern. Yet, few of these studies specifically addressed the issues with young children, and it is likely that behavior problems may emerge earlier than school age.

\section{Young children with developmental delays}

Given the rate of psychopathology in older populations of people with mental retardation, research has begun to explore the risk and protective factors associated with the development of dual diagnosis. To this end, young children identified as developmentally delayed or at risk for developmental delay have been studied. In one such study, Baker et al. (2002) reported an increased rate of behavior problems in children with developmental delays as compared to typically developing children at 3 years of age. Children were classified as having a developmental delay based on a score of less than 70 on the Bayley Scales of Infant Development. Twenty-six percent of the developmentally delayed children in this sample were reported by their parents to be in the clinical range for externalizing problems on the Child Behavior Checklist (CBCL). In contrast, only $8 \%$ of the typically developing children scored in this range, indicating that children with developmental delays were 3 times as likely to be in the clinical range for problem behaviors as early as age 3 years. When problem behaviors were again assessed 1 year later, children with developmental delays showed significantly more problem behaviors at age 4 years, and greater stability of problem behaviors than did typically developing children (Baker et al., 2003).

Studying even younger children, Feldman, Hancock, Rielly, Minnes, and Cairns (2000) assessed problem behaviors in 2-year-olds identified as developmentally delayed or at risk for developmental delays. Not all of these children had been formally diagnosed, but all were referred by agencies working with chil- dren with delays. Forty-two percent of parents reported some problem behaviors and $25 \%$ of the parents rated their children within the clinical range for behavior problems. Of interest, however, was the fact that this percentage was not significantly different than that reported in the normative sample of typically developing children. Although the percentage of children rated in the clinical range for broadband externalizing and internalizing problems was not elevated for the developmentally delayed sample, the 6 narrowband subscales of the CBCL were elevated. The authors suggested that children with developmental delays may show elevated risk for problem behaviors as young as 2 years, but that these problems are not sufficient to create more generalized externalizing or internalizing disorder. Nonetheless, Feldman et al. (2000) concluded that the relationship between delay and problem behaviors might develop over time as the child's interactions become more dependent on communication with others.

Preschool-age children identified as developmentally delayed have also been found to evidence greater problem behaviors and greater social skills deficits than their typically developing peers as rated by either a parent or teacher (Merrell \& Holland, 1997). It is important to note, however, that developmental delay in this study was a broad category that was not based on a formal assessment and included social-emotional delays as well as cognitive delays. Despite the problematic heterogeneity in the sample, this study adds to the growing base of evidence that suggests that children with early developmental delays show indications of significant behavior and social skill problems as early as the preschool years. Guralnick (1999) further presents a cogent discussion of the difficulties often found in peer-related social competencies of children with early developmental delays. Across multiple studies, Guralnick has shown that children with developmental delays engage in less interactive and more solitary play (Guralnick \& Groom, 1987), are more negative and difficult 
when conflicts with peers arise (Guralnick et al., 1998), and are less successful in gaining positive peer response (Guralnick, Connor, Hammond, Gottman, \& Kinnish, 1996). Such problems in peer social competencies are associated with adjustment difficulties for these children (Guralnick, 1999).

It seems apparent that the degree of developmental delay is also an important consideration in the types of behavior problems that children have. In a review of preschoolers' records from an inpatient unit (Johnson, Lubetsky, \& Sacco, 1995), child diagnoses were compared across a range of intellectual functioning. Sixty percent of the total sample met criteria for mental retardation. Comparing children across a range of intellectual functioning, those children classified in the borderline and mild mental retardation group were more likely to be diagnosed with ADHD or oppositional defiant disorder, while children in the lower range of intellectual functioning were most likely to be diagnosed with stereotypies. The authors attribute some of the difference in diagnosis rates to the nature of oppositional behavior, which requires a higher degree of cognitive functioning. Stereotypic behavior, in contrast, requires no higher order cognitive planning and therefore is more likely to be associated with more severe cognitive deficit in younger children. Other studies as well offer support that higher rates of behavior problems are found in children with more mild than severe delays, and the most typical behavior problems found are conduct disorders, anxiety disorders, and ADHD (Borthwick-Duffy \& Eyman, 1990; Grizenko, Cvejic, Vida, \& Sayegh, 1991; Jacobson, 1990). Despite this evidence, findings that greater behavior problems are associated with milder rather than more severe developmental deficits are not uniform (Koller, Richardson, Katz, \& McLaren, 1983; Myers, 1987). However, the research that suggests that behavior problems increase with more severe developmental deficits typically include data from adults with mental retardation and, therefore, are less directly relevant to considerations with young children.
Related to issues of specificity in degree of delay, questions are beginning to be raised regarding the specificity of type of developmental delay, particularly when the type involves some biopathological origin. As discussed earlier, Stromme and Diseth (2000) addressed this distinction when they differentiated psychopathology that was considered comorbid to the child's developmental delay from that which reflected a dominant psychopathology. Chadwick, Piroth, Walker, Bernard, and Taylor (2000) recently reported on risk factors for behavior problems in children with low intellectual functioning assessed using the Vineland screener. They found that both age and ambulation were important correlates. Younger delayed children had somewhat more severe problems, whereas ambulation was related to the types, but not severity of behavior problems reported. Children with lower levels of functioning (more organic in origin) were more likely to show destructive behavior, overactivity, self-injury, and sleeping difficulties, whereas physical aggression, attentionseeking behavior, and temper tantrums were not related to level of functioning. General self-help skills deficits were found to be as related to the development of behavior problems as were communication deficits in these children. What is intriguing is that neither sex of child nor socioeconomic status proved to be significant risk factors, leading Chadwick et al. (2000) to conclude that for children with intellectual delay, the neurological damage and severity of basic skills deficits may well override the effects of other risk factors typically found children with no developmental delays. It is difficult to discount the biological component in the emergence of behavior problems in young children, and it should not be discounted. However, it is interesting that those children with less delay, and therefore less clear organicity, are often the children with more behavior problems.

\section{Behavioral phenotypes}

Recently, Dykens and Hodapp have suggested that the risk of psychopathology for children with developmental delays is best 
understood by distinguishing among specific syndromes, rather than by treating children with developmental delay as a heterogeneous group (Dykens, 2000; Dykens \& Hodapp, 1997). Their research has shown that different psychiatric disorders are more prevalent with certain syndromes. For example, boys with fragile $\mathrm{X}$ syndrome are at increased risk for social anxiety, autism/pervasive developmental disorder, hyperactivity, and inattention, whereas girls with this disorder appear to be at increased risk for depression. In contrast, children with Down syndrome have a lower rate of comorbid psychopathology relative to children with undifferentiated developmental delays. Chadwick et al. (2000) likewise found that children with Down syndrome showed less severe behavior problems. Although the rates are lower, children with Down syndrome who do meet criteria for a psychiatric disorder are most often diagnosed with ADHD, oppositional, and anxiety disorders-problems which are similar to those in the population of young delayed children overall. Although the behavior problems for children with specific syndromes often overlap, suggesting that the behavioral phenotypes lack specificity, Dykens and Hodapp (1997) do note that distinguishing among the syndromes can be an important guide to treatment interventions that are tailored to specific children and their disability, and that clinicians need to take note of specific syndromes.

\section{Summary of behavior problems status}

It seems clear that children with developmental disabilities have a greater risk for behavior problems than do children who are typically developing, and children with mild delays are at somewhat greater risk overall. The full range of behavior disorders may be found in these children, and even young children show an array of difficulties that include oppositionality, anxiety, ADHD, and poor peer-related social competencies. Children with mild delays appear to be at greatest risk, as these children have the cognitive abilities to avail themselves of wider behavior choices, and individual differences in children remain important in determining the degree and extent of the behavior problems that surface. Although behavioral patterns characteristic of children with specific syndromes are growing in their popularity as explanations of problematic behavior, there are still a number of exogenous and endogenous factors that may be critical to the emergence of behavior problems early in children's lives. Chief among these factors are family functioning and newly emerging notions regarding children's regulatory competencies.

\section{FAMILY PROCESSES AND THE EMERGENCE OF BEHAVIOR PROBLEMS}

Given the significance of dual diagnosis as a clinical problem, the identification of risk factors for the emergence of behavior problems in children with developmental delays is of clear importance. In delineating these risk factors, both endogenous and exogenous factors would appear to be crucial considerations. Endogenous factors are those that reflect characteristics of the child and the developmental disorder itself (eg, temperament, neurobiological deficits associated with cognitive delay). Exogenous factors are those that represent influences external to the child, such as related family stress and constrained social networks and support systems. Despite the fact that research has continued to focus on single factor explanations of primary causal significance, current conceptualizations of dual diagnosis are beginning to acknowledge the complex interactive role that both exogenous and endogenous factors are likely to play.

The emergence of behavior problems in young children with developmental delays reflects an intricate transaction of factors merging across time. These factors likely include the endogenous indices of children's biologic condition that creates the developmental disability itself, and the child's temperamental reactivity. Exogenous factors of family functioning and parent-child relationships are also key determinants across time. Endogenous and exogenous factors combine to affect 
children's ability to regulate their behavior in the face of social and emotional challenge or ambiguity. Given decreased cognitive capacities, these young children struggle to adapt to the demands of emotionally and behaviorally challenging events, and the risk for behavior problems increases. These critical self-regulatory abilities include both emotional and behavioral facets (Eisenberg \& Fabes, 1992). Emotion regulation involves extrinsic and intrinsic management of the intensive and temporal features of emotional expressiveness and arousal, whereas behavior regulation refers to the adoption and internalization of a set of standards to manage one's behavior (Kopp, 1992).

Although this conceptual model has been articulated in depth elsewhere (Crnic, 2001; Crnic, Baker, Blacher, \& Edelbrock, 1998), a summary may help clarify the nature and the importance of the relations discussed above. The model is graphically presented in Figure 1. In this model, the presence of early developmental delay is strongly associated with various aspects of the family climate and parent-child relationships. Family climate and relationships are key determinants of children's developing self-regulatory behavior, which in turn influence the emergence of behavior problems and/or social competencies. Note, however, that we have not proposed a single pathway of influence. Rather, we have suggested a complex model of partial mediation in which the influence of developmental delay on children's behavior problems is mediated both by family processes and children's regulatory capacities. However, neither family processes nor children's ability to selfregulate completely accounts for problematic outcome, and the underlying biology associated with the developmental delay itself maintains some predictive value. Too, children's regulatory capacities do not entirely mediate family processes and context. Each element in the model maintains some direct association with emerging behavior problems. While we are currently engaged in a long-term, multisite, longitudinal study to examine this conceptual model (Crnic et al., 1998), a wealth of research exists to support the underlying premises of the model described, and merits some discussion.

\section{Biologic factors}

Towbin (1989) has argued that although individuals with developmental delays are susceptible to negative environmental effects, the emergence of behavior problems in these individuals has a primarily organic etiology. Towbin (1989), in fact, contends that neonatal cerebral damage is responsible for both intellectual deficits and behavioral disorders. Other research has suggested that because severity of intellectual disability is related to both the probability of an organic basis as well as the likelihood of psychopathology, psychopathology in this population is most likely to have an organic basis (see for review, Lacharite, Boutet, \& Proulx, 1995). Nonetheless, Lacharite et al. point out that other research that has studied individuals with developmental delays with and without neurological impairments has found no difference in the prevalence of psychiatric disorders

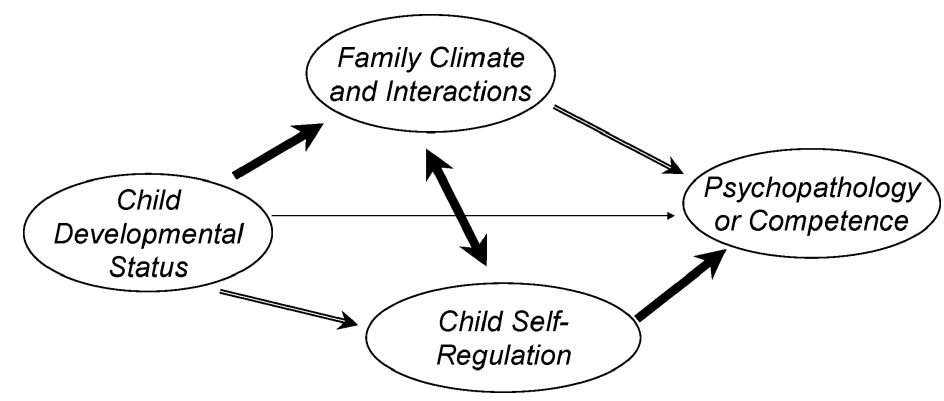

Figure 1. Model of dual diagnosis and behavioral competence. 
between the two groups. At this point, the full contribution of expressly biological explanations is unclear. Certainly, the work of Dykens and Hodapp (1997), identifying specific behavioral phenotypes of specific syndromes, suggests that underlying biological factors are important, but they do not tell the full story.

Beyond the expressly biologic factors, other endogenous factors have received attention as potential risk factors for the emergence of psychopathology, including child temperament. Although the vast majority of research on temperament has focused on children without developmental delays, Chess and Korn (1970) found that children with developmental delays who had certain temperament profiles characteristic of a more "difficult" temperament were much more likely to exhibit behavior problems than those with an "easy" temperament. Difficult temperaments, for example, might reflect characteristics in which children are less adaptive, more emotionally intense, and less sociable. However, in studies where temperament is thought to play a key role in the emergence of psychopathology, temperament is not generally thought to exert its influence directly. Rather, temperament is thought to affect behavior problems indirectly through its influence on other exogenous factors, such as the interactions between the child and his environment. Specifically, temperamental styles during early childhood are thought to help shape interactions within the family system, such that children with more difficult temperamental styles pose greater challenges to parenting. In cases for which parenting is already compromised, as is sometimes the case for young children with developmental delays, interactions between a child with a difficult temperament and parents who lack effective parenting skills are more likely to be characterized by a coercive pattern that can contribute to the development of childhood aggression and psychopathology (Patterson, 1986).

\section{Parent-child interactions}

Research examining parent-child interactions in families with children with devel- opmental delays has noted that these children pose unique parenting challenges that include intensified behavioral management issues (Baker, Blacher, Kopp, \& Kraemer, 1997). Given that these increased demands on parenting may overtax parenting resources and leave parents feeling ineffective, one might expect that, over time, coercive exchanges like those described above might ensue. These exchanges might then predispose children with developmental delays to develop behavioral disorders, resulting in the greater prevalence of behavioral disorders among this group of children than in the general population. Floyd and Phillippe (1993) tested this assumption, noting that children with developmental delays have been shown to be less compliant and, therefore, pose a greater challenge to parents' behavior management skills than do children without these delays. They found that, although the children with developmental delays had significantly higher behavior problem scores than children without delays, there were no significant differences in the rate of coercive exchanges in the two groups of families. Parents of children with delays were found to issue more commands during behavior management; however, these parents were also more likely than parents of children without delays to deliver a clear directive rather than a vague request. Floyd and Phillippe interpret these results as indicating that although children with mental retardation pose greater challenges to parents' behavior management skills, these parents are able to provide needed directives without falling into a coercive pattern of interaction. A later study of family problem solving again found that although parents had to make use of a greater number of directives to manage the behavior of children with delays, they did not fall into greater aversive behaviors than did parents in the comparison group (Costigan, Floyd, Harter, \& McClintock, 1997).

One possible explanation for the absence of coercive processes, despite the presence of increased behavior problems in families of children with delays, may be taken from the results of a study by Floyd and Zmich (1991). 
These investigators found a relation between poor perceived parenting skill and increased behavior problems among the comparison families, but not among those with a child with developmental delays. Floyd and Zmich (1991) subsequently reason that parents of children with developmental delays may be more likely to view behavior problems as related to endogenous child factors than parenting failures because they expect their children to display negative behaviors related to their disability. On the other hand, they also explain that parents of the nondelayed children may view these behaviors as unexpected and abnormal, and, therefore, may be more likely to attribute them to failures in parenting skill than to child factors.

\section{Family stress}

Despite the apparent resiliency in avoiding coercive behavior exchanges in the face of greater behavior management challenges, families of young children with developmental delay evidence other potential risk factors. For example, research has consistently demonstrated that families of children with developmental delays experience heightened levels of stress, particularly related to child rearing, which is not surprising considering the unique challenges these children present to parents (Crnic, Friedrich, \& Greenberg, 1983). Certainly, it has been well established that stress is related to behavior problems in both developmentally delayed and nondelayed samples of young preschool children (Baker \& Heller, 1996). Research has also indicated that parenting stress is an important predictor of children's behavior problems regardless of cognitive functioning (Crnic \& Greenberg, 1990; Heller, Baker, Henker, \& Hinshaw, 1996). However, given the typically higher levels of stress in families of children with developmental delays, stress can be expected to play an especially significant role in emerging behavior problems. For example, Baker et al. (2002) reported corroborating evidence that parents of children with developmental delays were more stressed than parents of children without delays. But Baker et al. also reported that in predicting parent stress in parents of children with developmental delays, child behavior problems accounted for significantly more of the variance than the children's level of cognitive functioning. Further, cognitive functioning contributed nonmeaningfully when behavior problems were entered first in regression analyses, implying that behavior problems rather than cognitive level per se play the key role in parents' stress levels. A second study by Baker et al. (2003) examined the relations between parenting stress and behavior problems over time in families with children with developmental delays. This study attempted to delineate the direction of effect in the relations between behavior problems and parenting stress in families of children with early developmental delays. They found that behavior problems were both predictive of and predicted by parents' stress, indicating that the higher instance of behavior problems in children with early developmental delays may be functionally related to early family stress and subsequently be associated with higher levels of family stress at later periods. This suggests that the development of behavior problems in children with early developmental delays is likely the result of a complex transactional process that includes child characteristics that challenge parents as well as factors that can compromise parenting, thereby adversely affecting children's behavior (Crnic, 2001).

\section{Child regulatory ability}

Given that both child and family characteristics are likely to influence the emergence of psychopathology in children with and without developmental delays, a crucial question arises: How do these various endogenous and exogenous factors together affect the development of disordered behavior? One possible connector involves the important role that self-regulation, and especially emotion regulation processes, plays in relation to the emergence of psychopathology or competence in children (Cole, Michel, \& Teti, 1994). Both child and family factors play key roles in the development of children's emotion regulation 
abilities (Brenner \& Salovey, 1997; Calkins, 1994). Although there are multiple perspectives on emotion regulation, and the construct is somewhat controversial, Cole et al. (1994) define emotion regulation as an individual's ability to respond to the ongoing demands of daily experiences with a range of emotions that are socially tolerable and sufficiently flexible to allow or inhibit spontaneous reactions. Cole et al. note that these abilities are important to success in a wide range of developmental tasks, such as being able to cope with frustration, maintain interest in learning, and adaptively engage in social relations with others. By contrast, emotion dysregulation would occur when established emotional patterns interfere with adaptive functioning across these domains. Cole et al. describe dysregulated emotional patterns as providing the basis for the development of disorder in terms of overregulated or underregulated behavior. Effective, as opposed to deficient, regulatory abilities are likely to have important consequences for psychological functioning, especially given the consistency of an individual's patterns of emotion regulation over time (Murphy, Eisenberg, Fabes, Shepard, \& Guthrie, 1999).

The ability to regulate one's own emotions develops as a function of both an infant's own abilities to self-regulate, as well as the abilities of the caregiver to recognize the infant's needs for assistance with regulatory functions (Brenner \& Salovey, 1997; Calkins, 1994; Shaw, Keenan, Vondra, Delliquadri, \& Giovannelli, 1997). Calkins (1994) points out that opposing parenting styles, such as responsive versus insensitive and accepting versus neglecting parenting, are likely to interact with children's behavior traits, such as adaptability versus reactivity, in order to promote or interfere with the development of emotion regulation abilities. When a caregiver is insensitive or unresponsive to an infant's needs, the infant's emotional state is likely to become dysregulated. Under these circumstances, the infant is obligated to devote coping resources to regulating negative affect, which limits the resources available to support the achievement of important developmental goals (Weinberg \& Tronick, 1998). In addition, infants may be so reactive to their environments that their caregivers are unable to be responsive to all of their distress, creating a greater regulation challenge for parents (Calkins, 1994).

Given the importance of both child and family factors in the development of emotion regulation competencies, children with developmental delays may be particularly susceptible to emotion regulation deficits for a variety of reasons. First, parents of children with developmental delays who are experiencing heightened levels of stress may be less available to aid in regulatory concerns. Second, intellectual deficits are likely to interfere with the acquisition of emotion regulation abilities. For example, Brenner and Salovey (1997) point out that children use emotional knowledge to help them with emotion regulation, including the ability to talk about emotions, determine others' emotional states, describe the experience of simultaneous emotions, and internalize cultural rules about emotional expressiveness. Clearly, children with compromised cognitive abilities would be more likely to experience difficulties with these tasks. Calkins (1994) also lists cognitive components such as the development of internal working models, the awareness of the need for regulation, and the ability to apply strategies as important sources of individual differences in emotion regulation abilities. Further, Brenner and Salovey (1997) describe the progression of emotion regulation strategies as moving from reliance on purely behavioral strategies toward the use of more cognitive strategies, such as cognitive distraction, which may not be as readily available to individuals with developmental delays. Brenner and Salovey also point out that children's ability to regulate emotions without assistance improves with age, which may be a much slower process for young children with developmental delays who may acquire these abilities at much slower rates or rely on different strategies altogether.

Few studies have examined emotion regulation difficulty in children with developmental 
delays, and its potential role in the emergence of dual diagnosis. In the only study to date, Wilson (1999) examined social entry behaviors in children with and without developmental delays and found that the delayed children utilized less effective emotion regulation strategies to cope with entry failure. This important, yet limited study is suggestive; however, more research is needed to assess the role that emotion regulation plays in the development of behavior problems in children with developmental delays. Further, research needs to address how specific child and parent characteristics in families of children with developmental delays interact to support or inhibit the development of effective emotion regulation abilities.

\section{Summary of factors affecting emerging behavior problems}

A wealth of data has established that families are affected by and affect their young children with developmental delays. These families are more stressed as a rule, and may engage in interactions that can place their child at further risk for developmental problems. Both stress and less positive interactions have been found to be correlates of behavior problems in children regardless of cognitive functioning, but clearly create increased risk in children that do have cognitive deficits. But family factors alone do not offer a complete explanation for the behavior problems, because child characteristics, such as temperament and self-regulatory ability, are likely key, and may in fact mediate the relations between developmental delay, family functioning, and the emergence of behavior problems during early childhood.

It is critical to note that the presence of early developmental delay is not a sentence to poorer outcomes across the various domains that have been discussed. Many children and families have resiliencies that have been well documented in the literature and the field has thankfully moved beyond the pathological models that so dominated thinking prior to the 1980s. However, it is important to be aware that the greater risk for behavior prob- lems in children with developmental delays is real and substantial. These behavior problems are not first apparent in adolescence or adulthood for persons with developmental disabilities, or even during middle childhood. Rather, problems are apparent as young as age 2 and 3 , and the factors associated with the emergence of these problems must be addressed at this early childhood period. Why is this critical? Because these behavior problems have important clinical costs. In the extreme, Blacher, Hanneman, and Rousey (1992) have noted that behavior problems are the major reason that families place their children with developmental disabilities out of home.

\section{IMPLICATIONS FOR PRACTICE}

The review and discussion provided above suggests that there is a need for practitioners to be aware that behavior problems are relatively common in young children with early developmental delays, even though such problems are not ubiquitous. When children with early developmental delays are identified, the potential for dual conditions (developmental delay and behavior disorder) should be a priority concern of professionals, and attention to children's behavioral status as well as their specific developmental/cognitive functioning should be the key foci of assessment.

Attention to the emergence of behavior problems in children with early developmental delays requires that professionals specifically address issues of family functioning, parent-child interactions, as well as children's temperamental functioning and emerging self-regulatory capacities. Each of these factors appears to represent important factors that contribute to mental health outcomes for these children, especially during the critical infancy and preschool period. In particular, the degree to which families are stressed requires attention, as stress is more common in families in which there is a child with a developmental delay. Family and parenting stresses are major predictors of subsequent adjustment difficulties for children (Crnic \& Low, 
2002), and stress typically serves as a change agent within families creating problematic circumstance. Families under stress require coping resources, such as social supports and facilitated access to appropriate services, to help prevent the kinds of changes that lead to adjustment problems in the children. Likewise, helping parents focus on the major interaction qualities that promote healthy adjustment is key. Sensitivity and positivity in interactions facilitate adjustment, whereas intrusiveness and negativity tend to be routinely associated with more problematic development. Parental directiveness is sometimes considered detrimental for typically developing children, but may be helpful for children with developmental delays and may not reflect an insensitive approach to building relationships (Marfo, 1992). Further, facilitating appropriate parent-child interaction will help support the emergence of stronger selfregulatory skills in young children with developmental delays, which should prove especially helpful in minimizing problem behavior in these children. It is critical to again caution that risk is not reality, and many children with early developmental delays do not evidence the kinds of behavior problems that have been discussed above. Nonetheless, it is imperative for professionals to be aware of the risks, attend to the various family and child considerations raised, and make appropriate referrals for specialized psychological services when concerns are present.

\section{REFERENCES}

Baker, B. L., Blacher, J., Crnic, K., \& Edelbrock, C. (2002). Behavior problems and parenting stress in families of three-year old children with and without developmental delays. American Journal on Mental Retardation, 107, 433-444.

Baker, B. L., Blacher, J., Kopp, C. B., \& Kraemer, B. (1997). Parenting children with mental retardation. International Review of Research in Mental Retardation, 20, 1-45.

Baker, B. L., \& Heller, T. L. (1996). Preschool children with externalizing behaviors: Experience of fathers and mothers. Journal of Abnormal Child Psychology, 24, 513-532.

Baker, B. L., McIntyre, L. L., Blacher, J. B., Crnic, K., Edelbrock, C., \& Low, C. (2003). Preschool children with and without developmental delay: Behavior problems and parenting stress over time. Journal of Intellectual Disability Research, 47, 217-230.

Blacher, J., Hanneman, R., \& Rousey, A. M. (1992). Out-ofhome placement of children with severe handicaps: A comparison of approaches. American Journal on Mental Retardation, 96, 607-616.

Borthwick-Duffy, S. (1994). Epidemiology and prevalence of psychopathology in people with mental retardation. Journal of Consulting and Clinical Psychology, 62(1), 17-27.

Borthwick-Duffy, S. A., \& Eyman, R. K. (1990). Who are the dually diagnosed? American Journal on Mental Retardation, 94, 586-595.

Brenner, E. M., \& Salovey, P. (1997). Emotion regulation during childhood: Developmental, interpersonal, and individual considerations. In P. Salovey \& D. J. Sluyter (Eds.), Emotional development and emotional in- telligence: Educational implications (pp. 168-195). New York: BasicBooks.

Calkins, S. D. (1994). Origins and outcomes of individual differences in emotion regulation. Monographs of the Society for Research in Child Development, 59, 5372.

Chadwick, O., Piroth, N., Walker, J., Bernard, S., \& Taylor, E. (2000). Factors affecting the risk of behavior problems in children with severe intellectual disability. Journal of Intellectual Disability Research, 44(2), 108-123.

Chess, S., \& Korn, S. (1970). Temperament and behavior disorders in mentally retarded children. Archives of General Psychiatry, 23, 122-130.

Cole, P. M., Michel, M. K., \& Teti, L. O. (1994). The development of emotion regulation and dysregulation: A clinical perspective. Monographs of the Society for Research in Child Development, 59, 73-100.

Costigan, C. L., Floyd, F. J., Harter, K. S. M., \& McClintock, J. C. (1997). Family process and adaptation to children with mental retardation: Disruption and resilience in family problem-solving interactions. Journal of Family Psychology, 11, 515-529.

Crnic, K. (2001, March). Family, emotion, and regulation: Process in the emergence of dual diagnosis. Invited address at the Gatlinburg Conference on Mental Retardation and Developmental Disabilities, Charleston, SC.

Crnic, K., Baker, B., Blacher, J., \& Edelbrock, C. (1998). Children with MR: Families, processes, and dual diagnosis. Research Grant (HD34879) funded by NICHD, NIH.

Crnic, K. A., Friedrich, W. N., \& Greenberg, M. T. (1983). 
The adaptation of families of mentally retarded children: A model of stress, coping, and family ecology. American Journal of Mental Deficiency, 88, 125138.

Crnic, K. A., \& Greenberg, M. T. (1990). Minor parenting stresses with young children. Child Development, 61, 1628-1637.

Crnic, K. A., \& Low, C. (2002). Everyday stresses and parenting. In M. Bornstein (Ed.), Handbook of parenting (Vol. 4, 2nd ed, pp. 243-268). Hillsdale, NJ: Erlbaum.

Dykens, E. (2000). Psychopathology in children with intellectual disability. Journal of Child Psychology and Psychiatry, 41, 407-417.

Dykens, E. M., \& Hodapp, R. M. (1997). Treatment issues in genetic mental retardation syndromes. Professional Psychology Research and Practice, 28, 263270.

Eisenberg, N., \& Fabes, R. (1992). Emotion, regulation, and the development of social competence. In M. S. Clark (Ed.), Emotion and social behavior: Review of personality and social psychology (Vol. 14, pp. 119150). Newbury Park, CA: Sage.

Feldman, M., Hancock, C., Rielly, N., Minnes, P., \& Cairns, C. (2000). Behavior problems in young children with or at risk for developmental delay. Journal of Child and Family Studies, 9(2), 247-261.

Floyd, F. J., \& Phillippe, K. A. (1993). Parental interactions with children with and without mental retardation: Behavior management, coerciveness, and positive exchange. American Journal on Mental Retardation, 97, 673-684.

Floyd, F. J., \& Zmich, D. E. (1991). Marriage and parenting partnership: Perceptions and interactions of parents with mentally retarded and typically developing children. Child Development, 62, 1434-1448.

Grizenko, N., Cvejic, H., Vida, S., \& Sayegh, L. (1991). Behaviour problems in the mentally retarded. Canadian Journal of Psychiatry, 36, 712-717.

Guralnick, M. J. (1999). Family and child influences on the peer-related social competence of young children with developmental delays. Mental Retardation and Developmental Disabilities Research Reviews, 5, 2129.

Guralnick, M. J., Connor, R., Hammond, M., Gottman, J. M., \& Kinnish, K. (1996). Immediate effects of mainstream settings on the social interactions and social integration of preschool children. American Journal of mental Retardation, 100, 359-377.

Guralnick, M. J., \& Groom, J. M. (1987). Dyadic peer interactions of mildly delayed and non-handicapped preschool children. American Journal of Mental Deficiency, 92, 178-193.

Guralnick, M. J., Paul-Brown, D., Groom, J. M., Booth, C. L., Hammond, M. A., Tupper, D. B., et al. (1998). Conflict resolution patterns of preschool children with and without developmental delays in heterogeneous playgroups. Early Education and Development, 9, 49-77.
Heller, T. L., Baker, B. L., Henker, B., \& Hinshaw, S. P. (1996). Externalizing behavior and cognitive functioning from preschool to first grade: Stability and predictors. Journal of Clinical Child Psychology, 25, 376387.

Jacobson, J. (1990). Do some mental disorders occur less frequently among persons with mental retardation? American Journal on Mental Retardation, 94(6), 596-602.

Johnson, C., Lubetsy, M., \& Sacco, K. (1995). Psychiatric and behavioral disorders in hospitalized preschoolers with developmental disabilities. Journal of Autism and Developmental Disorders, 25(2), 169182.

Koller, H., Richardson, S. A., Katz, M., \& McLaren, J. (1983). Behavior disturbance since childhood among a 5-year birth cohort of all mentally retarded young adults in a city. American Journal on Mental Deficiency, 87, 386-395.

Kopp, C. B. (1992). Emotion and its regulation in early development. New Directions for Child Development, 55, 41-56

Lacharite, C., Boutet, M., \& Proulx, R. (1995). Intellectual disability and psychopathology: Developmental perspective. Canada's Mental Health, 43, 2-8.

Marfo, K. (1992). Correlates of maternal directiveness with children who are developmentally delayed. American Journal of Orthopsychiatry, 62, 219233.

Matson, J., \& Frame, C. (1986). Psychopathology among mentally retarded children and adolescents. Beverly Hills, CA: Sage.

Merrell, K., \& Holland, M. (1997). Social-emotional behavior of preschool children with and without developmental delays. Research in Developmental Disabilities, 18(6), 393-405.

Murphy, B. C., Eisenberg, N., Fabes, R. A., Shepard, S., \& Guthrie, I. K. (1999). Consistency and change in children's emotionality and regulation: A longitudinal study. Merrill-Palmer Quarterly, 45, 413444.

Myers, B. A. (1987). Psychiatric problems in adolescents with developmental disabilities. Journal of the American Academy of Child and Adolescent Psychiatry, 26, $74-79$

Nezu, C., Nezu, A., Gill-Weiss, M. (1992). Psychopathology in persons with mental retardation: Clinical guidelines for assessment and treatment. Champaign, IL: Research Press.

Patterson, G. R. (1986). Performance models for antisocial boys. American Psychologist, 41, 432-444.

Pfeiffer, S., \& Baker, B. (1994). Residential treatment for children with dual diagnoses of mental retardation and mental disorder. In J. Blacher (Ed.), When there's no place like bome: Options for children living apart from their natural families. Baltimore, MD: Paul $\mathrm{H}$. Brookes.

Shaw, D. S., Keenan, K., Vondra, J. I., Delliquadri, E., 
\& Giovannelli, J. (1997). Antecedents of preschool children's internalizing problems: A longitudinal study of low-income families. Journal of the American Academy of Child \& Adolescent Psychiatry, 36, 1760-1767.

Steffenburg, S., Gillberg, C., \& Steffenburg, U. (1996). Psychiatric disorders in children and adolescents with mental retardation and active epilepsy. Archives of Neurology, 53, 904-912.

Stromme, P., \& Diseth, T. (2000). Prevalence of psychiatric diagnoses in children with mental retardation:
Data from a population-based study. Developmental Medicine and Child Neurology, 42, 266-270.

Towbin, A. (1989). Behavior disorders in mentally retarded persons, the dually diagnosed: Factors of pathogenesis. Journal of Clinical Psychology, 45, 910-918.

Weinberg, M. K., \& Tronick, E. Z. (1998). The impact of maternal psychiatric illness on infant development. Journal of Clinical Psychiatry, 59, 53-61.

Wilson, B. J. (1999). Entry behavior and emotion regulation abilities of developmentally delayed boys. Developmental Psychology, 35, 214-222.

Lists of current articles: http:/ / depts.washington.edu/ isei/ iyc/ iyc_comments.html

To view previous articles: http:/ / depts.washington.edu/ isei/ iyc/ iyc_previous.html 